\title{
Constitutivity in Flavour Perception
}

\author{
Błażej Skrzypulec ${ }^{1}$ (1)
}

Received: 5 October 2020 / Accepted: 3 December 2021

(c) The Author(s) 2021

\begin{abstract}
Within contemporary philosophy of perception, it is commonly claimed that flavour experiences are paradigmatic examples of multimodal perceptual experiences. In fact, virtually any sensory system, including vision and audition, is believed to influence how we experience flavours. However, there is a strong intuition, often expressed in these works, that not all of these sensory systems make an equal contribution to the phenomenology of flavour experiences. More specifically, it seems that the activities of some sensory systems are constitutive for flavour perception while others merely influence how we experience flavours. This paper aims to answer the question regarding the constitutive factors of flavour perception in a twofold way. First, a theoretical framework is developed, relying on debates regarding constitutivity in analytic metaphysics and philosophy of science, which defines the stronger and weaker senses in which the activities of sensory systems may be constitutive for flavour perception. Second, relying on empirical results in flavour science, the constitutive status of activities related to distinct sensory systems in the context of flavour perception is investigated.
\end{abstract}

\section{Introduction}

Within contemporary philosophy of perception, it is commonly claimed that flavour experiences are paradigmatic examples of multimodal perceptual experiences (e.g., Smith, 2013; Stevenson, 2014). Typically, flavour experiences occur due to the activities of various sensory systems processing, inter alia, gustatory, olfactory, tactile, thermal and trigeminal information (see Delwiche, 2004; Verhagen \& Engelen, 2006). In particular, the narrowly understood sense of taste, which processes merely stimuli interacting with chemical receptors on the tongue, cannot give rise to the majority of typical flavour experiences such as experiences of strawberry or coffee flavours (e.g., Auvray \& Spence, 2008; Spence et al., 2014). In consequence, it is

Błażej Skrzypulec

blazej.skrzypulec@uj.edu.pl

1 Institute of Philosophy, Jagiellonian University, ul. Grodzka 52, 31-044 Kraków, Poland 
postulated that taste, or more technically gustation, should be understood as one of the unimodal components of a multimodal flavour sense.

In fact, virtually all sensory systems, including vision and audition (e.g., Carvalho et al., 2017; Spence, 2014; Spence, 2015c), are believed to influence how we experience flavours. However, there is a strong intuition, often expressed in philosophical works regarding flavour perception, that not all of these sensory systems make an equal contribution to flavour perception. More specifically, it seems that the functioning of some sensory systems is constitutive of flavour perception while others merely influence how we experience flavours. For instance, Smith (2013, p. 307) claims that "there is no agreement on what is necessary or constitutive of flavour experience" and postulates that factors which causally affect flavour perception should be distinguished from the constitutive factors. Similarly, Prescott (2015, p. 47) postulates a distinction between intrinsic constituents and extrinsic elements affecting flavour experiences, while Spence (2015c, p. 24) writes about the need to distinguish factors constitutive of flavour perception and factors that merely modulate flavour experiences.

The above examples demonstrate a need to distinguish constitutive and nonconstitutive components of flavour perception that has not yet been satisfied by philosophers of perception. From the philosophical perspective, addressing this need will require explicating what it means to say that some factors are 'constitutive' of flavour perception and providing a criterion for distinguishing constitutive and nonconstitutive factors. However, in contemporary philosophical and empirical works regarding flavour perception, the notion of 'constitution' is used in a loose sense, without stronger connections to the philosophical debate regarding constitutivity, and without providing criteria for assessing the constitutivity of sensory systems. This paper aims to fill in this theoretical gap by (a) explicating the notion of 'constitutivity' in the context of flavour perception relying on philosophical discussions, (b) distinguishing the stronger and weaker meanings of constitutivity, and (c) showing that the proposed conceptual framework can be used to assess the constitutivity of various sensory activities by relying on empirical results.

The specific issue addressed in this paper concerns the constitutive relation between the functioning of sensory systems and the ability of human flavour perception to generate flavour experiences. ${ }^{1}$ For instance, a relevant question is whether without the functioning of the visual system, some flavour experiences cannot be generated, or whether without the functioning of the olfactory system any flavour experiences can occur. The main intuition behind my conceptualisation of constitutiveness is that functioning of a sensory system is constitutive of flavour perception if some activities of this sensory system belong to minimal sets of conditions

\footnotetext{
1 A further question, which cannot be accommodated in the scope of this paper, concerns the relation between functioning of certain sensory systems and the presence of certain phenomenal aspects of flavour experiences. For instance, it may be proposed that touch is mainly relevant for phenomenal aspects determining spatial properties of flavour stimuli, while olfaction is for phenomenal aspects determining perceptual categorisation of eaten items.
} 
jointly sufficient for the occurrence of some flavour experiences (see Sect. 2 for explication).

I conduct my analyses in the context of normally functioning human flavour perception. In consequence, all further statements about the necessity of some sensory activities should be read in a restrictive way as applying only within the context of normally functioning human cognitive systems (see more on this in Sect. 3).

Given the above characterization of my approach, the notions of "flavour experiences" and "sensory systems" play a crucial role in the investigations conducted. I characterize flavour experiences following Auvray and Spence (2008), who describe flavour perception as a perceptual modality that represents the properties of entities positioned in the mouth in order to assess whether they can be eaten (see also Stevenson, 2009). ${ }^{2}$ From this perceptive, flavour experiences can be characterized as those which seem to occur in the mouth and which present properties of external objects.

Nevertheless, the above characterization has to be extended in order to distinguish flavour experiences from taste experiences, because even sensations occurring due to mere stimulation of gustatory receptors, like the sensation of bitterness, may be exteroceptive and seem to happen in the mouth. An initial proposition could be to claim that what distinguishes flavour experiences from taste experiences is multimodality: flavour experiences occur due to the activity of more than one sensory system, while taste experiences are unimodal. Nevertheless, even the paradigmatic taste experiences involve both some gustatory processing, due to which we may feel sensations like bitterness, and some tactile processing, due to which we localize the sensation in the mouth. To avoid this problem, I propose a modified approach according to which, flavour has to occur by virtue of the activity of at least two sensory systems such that the activities of each of these systems add something more to the phenomenal character ${ }^{3}$ of an experience than apparent localisation in the oral cavity.

However, even after the above addition the characterization of flavour experiences would be too wide. For instance, an experience associated with having a cold, tasteless plastic cube which seems to be occurring in the mouth is exteroceptive and multimodal by being produced by thermal and tactile senses, but intuitively it should not be considered as a flavour experience. Hence, in order to grasp the above intuition, an additional requirement has to be added, namely that flavour experiences are those which are commonly referred to by using terms naming flavour-categories (e.g., 'strawberry', 'vanilla', 'fishy').

Taking together the above observations, I propose the following definition of flavour experiences which will be used in the paper:

\footnotetext{
${ }^{2}$ In consequence, I adopt a kind of realism in regard to flavours, according to which they are understood as properties of external objects that are represented in flavour experiences (see Smith, 2013).

${ }^{3}$ I do not assume any particular theory of phenomenal character of sensory experiences. I use this term in accordance with a common philosophical intuition that phenomenal character is this aspect which determines "what is it like" to have a given experience. For instance, experiencing a sweet taste feels different from experiencing a sour taste and this difference consists in having distinct phenomenal characters.
} 
(Flavour Experience) An experience is a flavour experience if and only if (a) it seems to occur in the mouth, $(b)$ is an exteroceptive experience, (c) it occurs in virtue of the activity of at least two sensory systems such that the activities of each of these systems add something more to the phenomenal character of an experience than the apparent localisation in the oral cavity, and $(d)$ is commonly characterised by using predicates naming flavour-categories.

The above definition is sufficient for further investigation as its grasps the main intuitions connected with typical situations of experiencing flavours. ${ }^{4}$ For instance, there are olfactory experiences which are not flavour experiences due to lack of apparent localization in the mouth despite the fact that they are exteroceptive and are often characterized by terms naming flavour-categories (e.g., 'lemony' or 'vanilla'). Similarly, some experiences intuitively happening in the mouth, such as tooth pain, are not flavour experiences because they are interoceptive states representing states of a body, and not properties of external entities. An important advantage of the proposed definition is that it does not assume the constitutive character of any sensory system. In consequence, it is suitable for investigations concerning which senses are constitutive of flavour perception.

When using the notion of "sensory systems", I follow a physiological approach used in flavour-related literature (see Stevenson \& Tomiczek, 2007; Verhagen, 2007). ${ }^{5}$ According to this idea, distinct sensory systems use different groups of receptors to detect stimuli and initially process obtained information using separate neural mechanisms. For instance, olfactory and gustatory sensory systems gather information using receptors with differing structures and localizations. Subsequently, they analyse the obtained information using distinct neural pathways, and only later is the gathered information combined and jointly processed by common structures (Simons \& Noble, 2002; Small \& Prescott, 2005; Verhagen \& Engelen, 2006).

According to the physiological literature, there are many sensory systems whose activities are likely to contribute to the occurrence of flavour experiences. In the paper, I consider four such systems that are frequently discussed in empirical and philosophical works concerning flavour perception. These four systems are divided into two groups, on the basis of the strength of intuitions regarding the constitutive status of their functioning for flavour perception. The first group contains gustatory and olfactory systems. The functioning of these systems are unanimously treated as constitutive of flavour perception and virtually every characterization of flavour modality sees them as crucial for generating flavour experiences (see Auvray \& Spence, 2008). The second group encompasses visual and auditory systems. While

\footnotetext{
${ }^{4}$ It should be noted that despite the presence of point (d), my definition is coherent with a view that flavour experiences are purely perceptual experiences without a cognitive component. This is because they happen in virtue of the functioning of sensory systems. However, recognising which of the various perceptual experiences are flavour experiences may have a cognitive component which is included in my definition. Nevertheless, it does not entail flavour experiences being produced by higher-level, cognitive mechanisms.

5 For the complications and limitations of characterizing sensory modalities in physiological terms see Keeley (2002).
} 
many authors believe that the functioning of these systems influence flavour perception, it is doubtful whether it is constitutive (Smith, 2013; Spence \& Zampini, 2006; Velasco et al., 2018).

The paper starts by providing a notion of minimal constitutivity that is developed relying on intuitions presented in works regarding analytic metaphysics and philosophy of science (Sect. 2). Subsequently (Sect. 3), stronger notions of constitutivity are defined, and I explicate how they can be applied in considerations about flavour perception. Further, relying on empirical data, I consider the constitutive status of functioning of the olfactory and gustatory systems (Sect. 4), and visual and auditory systems (Sect. 5). The conducted analyses confirm the intuitively strong constitutive status of olfactory and gustatory systems. Furthermore, they also allow explicating the type of constitutivity possessed by these systems. On the other hand, it is argued, in contrary to common intuition, that auditory system is constitutive of flavour perception. Nevertheless, its constitutive status is of a weaker type than in case of olfactory and gustatory systems. Finally, it is shown that there are serious reasons to doubt whether functioning of visual system is constitutive at all.

\section{Minimal Constitutivity}

In my investigations concerning the constitutivity in flavour perception, I will rely on two philosophical discussions in which the notion of "constitution" plays a major role. The first is the metaphysical debate regarding material constitution (e.g., Bennett, 2011; Wasserman, 2004; Wilson, 2007). For instance, it seems that an iron monument is constituted by a lump of iron but is not identical to it (e.g. a lump of iron may still exist after the destruction of a statue), and the task of analytic metaphysics is to characterise the nature of such a constitution relation. Secondly, within the philosophy of science, the notion of "constitution" is crucial for investigations regarding constitutive mechanistic explanation. It is frequently postulated that some phenomena, for instance biological or psychological, are constituted by an underlying mechanism, and one goal of a scientific model is to explain a given phenomenon by describing this mechanism (e.g., Baumgarten and Gebharter, 2015; Craver, 2007; Kaiser \& Krickel, 2016; Krickel, 2018; Ylikoski, 2013). To provide a proper mechanistic explanation, a criterion is needed which allows us to distinguish the elements that constitute the relevant mechanism from those that merely causally influence its functioning.

In both these fields, authors postulate similar basic characteristic of the constitution relation. First, it is a synchronic relation, i.e. a relation occurring between entities existing at the same moment (e.g., Baumgarten and Gebharter, 2015; Kirchhoff, 2013). ${ }^{6}$ This characteristic is believed to distinguish constitution from causation, which, according to standard approaches, occurs between entities existing at distinct moments (e.g., Gillett, 2013; Leuridan, 2012; Ylikoski, 2013). Second, constitution is an interlevel relation that connects more fundamental entities (those which

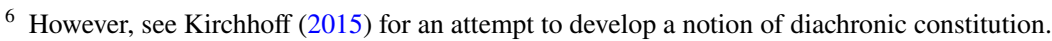


constitute) with less fundamental entities (those which are constituted). Third, constitution is a relation of asymmetric dependency, as the existence of constituted entities somehow relies on the existence of constituting entities (e.g., Bennett, 2011; Couch, 2011; Gebharter, 2016).

It seems plausible that the relation between some activities of sensory systems and flavour experiences satisfies these basic characteristics. This is because the relevant neural activities temporally overlap with the occurrence of flavour experiences and are considered more ontologically basic than phenomenal, mental states. Furthermore, flavour experiences depend on the activities of sensory systems as they at least supervene on neural activity.

The above basic intuitions concerning constitution are typically specified in two general ways. First, it is claimed that constitution requires not only temporal but also spatiotemporal overlap between constituting and constituted entities, or that constituting entities are parts of the constituted whole (see Bennett, 2011; Baumgarten and Gebharter, 2015; Kirchhoff, 2013). For instance, it seems plausible that to constitute a statue, a lump of iron has to be spatially co-located with a statue. If a statue were located at a place $P$ at a moment $T$ and a lump of iron were located at a disjoint place $G$ at $T$, it would be very unintuitive to allow that this lump can compose the considered statue at $T$. In subsequent parts of the paper, I do not investigate such mereological and topological aspects of constitution. The claim that activities of the sensory system are parts of flavour experiences or that they spatiotemporally overlap with such experiences requires accepting a specific theory regarding relations between mind and body. However, I do not assume any strong thesis concerning the resolution of the mind-body problem besides the general claim that flavour experiences supervene on neural activity.

The second popular way of explicating intuitions concerning constitution, which will be crucial in subsequent investigations, is specifying the dependency relation between constituting and constituted entities. Within works regarding the metaphysical problem of material constitution, it is claimed that a specific arrangement of constituting elements is sufficient for the presence of a constituted entity (see the 'intrinsic necessitation' condition in Wilson, 2007). For instance, if fragments of an iron lump are in an appropriate spatial relation, then a statue exists. On the other hand, a particular arrangement of constituting entities is usually not necessary for the existence of a constituted entity. For example, it seems that the same iron statue can survive some changes concerning the constituting material. When the iron lump constituting a statue loses some iron atoms, it becomes a distinct lump. However, despite those changes in the constituting material, it seems intuitive to state that the statue is not replaced by a distinct one but remains the same. Nevertheless, a particular statue clearly cannot be constituted by any metal lump, and so there seems to be a set of alternative, sufficient arrangements of constituting elements (see the 'constituent necessitation' condition in Wilson, 2007). While I intend to preserve the above intuitions in my approach to constitutivity, I do not simply adapt the notion of constitutivity used in discussions on the problem of material constitution, because in these considerations the notion of constitution usually assumes the presence of spatial overlap between constituting and constituted entities. 
Similar intuitions, but without a strong commitment to the problematic idea of spatial overlap, are expressed by philosophers of science who develop regularist notions of constitutive mechanistic explanation (see Couch, 2011; Harbecke, 2010). According to such positions, an activity $A$ of a mechanism's part is constitutive for a phenomenon $F$ if and only if the presence of $A$ is an element of a minimal set of conditions whose joint satisfaction is sufficient for $F$ 's occurrence. The minimality of a set means that without the satisfaction of any of its elements, for instance the condition concerning the presence of $A$, the satisfaction of the remaining conditions would not be, ceteris paribus, enough for the occurrence of the considered phenomenon $F$. In other words, the constitutivity of $A$ for $F$ entails that $A$ is a necessary element of a set of jointly sufficient conditions of $F$ 's occurrence. Nevertheless, a minimal set of sufficient conditions which includes the condition concerning the presence of $A$ does not have to be a set of conditions whose joint satisfaction is necessary for $F$ 's occurrence. It may be the case that there is a disjunction such that each its element is a minimal set of conditions jointly sufficient for the occurrence of $F$, no element of the disjunction is a set of jointly necessary conditions for $F$ 's occurrence, and the satisfaction of the whole disjunction is necessary for the presence of $F$.

The above approach to constitution expresses important intuitions about the logical relations between constituting and constituted entities. First, the presence of constituting entities, appropriately arranged, is sufficient for the occurrence of a constituted entity. Second, constituted entities have a minimal set of conditions, concerning the presence of constituting entities and relations between them, such that the joint satisfaction of these conditions entails the presence of a constituted entity. The minimality means that even a lack of satisfaction regarding one condition causes the satisfaction of the remaining conditions to be, ceteris paribus, insufficient for the occurrence of the constituted entity. Third, a single constituted entity may have many different minimal sets of jointly sufficient conditions concerning the presence of constituting entities and the relations between them. This means that there may be alternative ways in which a constituted entity is formed.

By applying the above the ideas proposed by Couch (2011) and Harbecke (2010) to the constitutivity problem concerning flavour perception, a notion of minimal constitutivity can be formulated which serves as a foundation for my further investigations. This notion describes a minimal condition that has to be satisfied by a sensory system in order to treat it as constitutive of flavour perception. According to this notion, the functioning of a sensory system $S$ is minimally constitutive of flavour perception if and only if there is a flavour experience $F$ such that the presence of an activity of $S$ is an element of a minimal set of conditions jointly sufficient for the occurrence of $F$. In addition, to satisfy the basic intuitions regarding constitution relations, the relevant activity of $S$ has to be synchronous with the occurrence of $F$. This does not necessarily mean that a sensory activity has to last from the beginning to the end of an experience, as many flavour experiences can occur by virtue of many subsequent sensory activities. However, a modest requirement for constitutivity is that constitutive activities have to at least temporally overlap with constituted experiences. 
In other words, minimal constitutivity of a system $S$ means that there is a flavour experience $F$ such that there is a way of obtaining it which requires the presence of an activity of system $S$. For instance, it may be the case that there is a way of obtaining an experience presenting a strawberry flavour which requires that the olfactory system is activated in a certain way. If this is the case, then functioning of olfactory system is minimally constitutive of flavour perception. In the next section, I characterise the notion of minimal constitutivity more precisely in the context of flavour experiences and formulate stronger types of constitutivity.

\section{Flavour Experiences and Varieties of Constitutivity}

The activities of human sensory systems contribute to the occurrence of a huge variety of distinct flavour experiences. Let's use the term "Flavour Experiences Class" to name a class whose members are all flavour experiences that can be produced by normally functioning, human sensory systems:

(FEC) Flavour Experiences Class is a class containing all flavour experiences, individuated by their phenomenal character, which can occur by virtue of activities of normally functioning human sensory systems.

FEC describes the space of possibilities available to human flavour perception. It should be noted that it is not necessarily the case that every human being has to be able to posses all elements of FEC. This is because there are significant individual differences related to the physiology of chemical receptors (see Bartoshuk et al., 1998 on the distinction between 'nontasters' and 'supertasters', and Reed \& Knaapila, 2010 for a review on genetic causes of individual differences), which may determine whether a person can have a certain flavour experience. Furthermore, flavour experiences often depend on a learned association between stimulations of the sensory systems relevant for flavour perception (see Rolls, 1997; Wan et al., 2014), and so available flavour experiences may vary with geographical and cultural context. What $F E C$ describes are not the abilities of some particular humans but rather the maximal abilities of human flavour perception. Furthermore, elements of FEC should not be treated as instantaneous experiences, as some flavour experiences may have a complex temporal structure (e.g., experiences associated with drinking wine are usually characterized as having temporal phases; see essays in Smith, 2007).

Despite its broad range, the extension of FEC is restricted by the normal functioning of human sensory systems. Here, 'normal' is understood quite widely, such that it encompasses the individual physiological differences and cultural differences mentioned above. What I want to exclude are, in particular, potential flavour experiences that may be obtained by directly stimulating the central nervous system without activating many of the usual sensory mechanisms and those which are caused by serious disturbances in the functioning of sensory and cognitive mechanisms. Elements of FEC are individuated by phenomenal character, in the intuitive sense that each element differs in terms of "what is it like" to have that experience.

The definition of FEC states that its members occur by virtue of the activities of human sensory systems. This means that for each experience $F$ belonging to FEC there is a minimal set of conditions jointly sufficient for the occurrence of $F$ such 
that some of these conditions concern the activities of sensory systems. It should be noted that such a minimally sufficient set of occurrence conditions (henceforth $M S S$ ) does not have to contain only conditions concerning sensory activities. For instance, occurrences of flavour experiences may also require the activity of some central mental mechanisms that cannot easily be associated with any sensory system (e.g., see Deroy et al., 2016 for a review on top-down, metacognitive influences on multisensory integration).

A single flavour experience may be associated with many MSSs. In such a case there is more than one way in which a given experience can be obtained. All MSSs associated with a single flavour experience $F$ constitute a disjunction such that its satisfaction is a necessary condition of $F$ 's occurrence. Furthermore, every condition belonging to any $M S S$ is its necessary element. This is because every MSS of some experience $F$ is a minimal set of $F$ 's jointly sufficient occurrence conditions and in consequence a lack of any of its elements would cause the joint satisfaction of remaining conditions to be, ceteris paribus, no longer sufficient for the occurrence of $F$.

Relying on the above remarks, we may provide a precise characterization of what it means, following regularist approach to constitution (Couch, 2011; Harbecke, 2010), to say that the functioning of some sensory system is minimally constitutive of flavour perception:

(Minimal Constitutivity) The functioning of a sensory system $\mathrm{S}$ is minimally constitutive of flavour perception if and only if there is an experience $\mathrm{F}$ belonging to FEC such that $\mathrm{F}$ has an MSS containing a condition concerning the presence of a synchronic activity of the system $\mathrm{S}$.

In other words, the functioning of a sensory system $S$ is minimally constitutive if, and only if, there is at least one flavour experience such that there is a way of obtaining it requiring the presence of some activity of $S .{ }^{7}$ For a more concrete example, let's consider Titchener's famous description of ripe peach flavour, which is experienced as a blend of, inter alia, "pucker feel of the sour", "softness and stringiness", and "ethereal odour" (Titchener, 1909, p. 135). ${ }^{8}$ If there is a way of obtaining such an experience that requires some activities of the olfactory system, then functioning of the olfactory system is minimally constitutive of flavour perception.

Minimal constitutivity is not the only type of constitutivity that can be defined using the above conceptual framework. In particular, a stronger version, named 'essential constitutivity', may be characterized as follows:

(Essential Constitutivity) The functioning of a sensory system $\mathrm{S}$ is essentially constitutive of flavour perception if and only if there is an experience $\mathrm{F}$ belonging to FEC such that all its MSSs contain a condition concerning the presence of a synchronic activity of the system $\mathrm{S}$.

\footnotetext{
7 If not mentioned otherwise, by activities I mean synchronic activities, i.e. those that at least temporally overlap with the considered flavour experience.

${ }^{8}$ Of course, this is a simplification, as 'ripe peach flavour experience' names a type of flavour experience and not a particular experience.
} 
Essential constitutivity means that there is no way to obtain a certain flavour experience without functioning of a sensory system $S$. In other words, if people did not possess system $S$, then $F E C$ would be different as it would not contain flavour experiences that cannot be obtained without the activities of $S$. For instance, it may be the case that the experience of ripe peach flavour cannot be produced without some activities of the olfactory system. If this is true, then olfactory system is essentially constitutive of flavour perception. Such dependence is not held by minimal constitutivity because in that case there is a way of obtaining a flavour experience that requires the functioning of $S$, but it may still be possible to obtain this experience without the functioning of $S$.

Furthermore, an additional, stronger variant of essential constitutivity can be formulated by considering that flavour experiences are commonly divided into types, usually named after an object that is associated with paradigmatic experiences of a given type. For instance, it seems that despite some phenomenal differences, the flavour experiences associated with drinking sweet coffee and drinking bitter coffee are treated as exemplars of the same coffee-type of flavour experience. It is very likely that borders between types are vague, as there may be differences, caused by individual and cultural factors, in how people classify the same flavour experiences (see Stevenson \& Boakes, 2004; Wan et al., 2014). Nevertheless, I believe that it is plausible to assume that there are some commonly perceived similarities between certain flavour experiences and so there will be some regularities in dividing experiences into types (see Smith, 2013). For example, it is very likely that people sharing a similar cultural background will assign experiences of sweet coffee and bitter coffee to one type.

If one accepts that at least some elements of FEC can be assigned to types, then a following stronger version of essential constitutivity can be formulated:

(Essential-type Constitutivity) The functioning of a sensory system $S$ is essentially-type constitutive of flavour perception if and only if there is a type of experiences belonging to FEC such that for every exemplar of this type all its MSSs contain a condition concerning the presence of a synchronic activity of the system $\mathrm{S}$.

Essential-type constitutivity is stronger than essential constitutivity. If the functioning of some sensory system $\mathrm{S}$ is essentially-type constitutive, then without this system not only can some flavour experiences not be obtained but a whole type of experiences would disappear from our flavour perception. For instance, it may be the case that without functioning of the olfactory system not only can flavour experiences of ripe peach not occur but the same is true of all peach-related flavour experiences, like experiences of peach ice-cream or peach yogurt.

Nevertheless, essential-type constitutivity is still not the strongest kind of constitutivity that can occur between the functioning of sensory systems and flavour experiences. It is possible that functioning of some sensory system is 'absolutely constitutive' in the following sense:

(Absolute Constitutivity) The functioning of a sensory system $\mathrm{S}$ is absolutely constitutive of flavour perception if and only if for every experience belonging to FEC it is the case that all its MSSs contain a condition concerning the presence of a synchronic activity of the system $\mathrm{S}$. 
If functioning of a sensory system $S$ is absolutely constitutive, then without this system no flavour experiences could be obtained, or in other words $F E C$ would be an empty class. For example, if olfactory system is absolutely constitutive of flavour experiences, then we not only cannot experience peach flavour without olfaction, but literally no flavour experience can occur without the functioning of the olfactory system.

As it is easy to see, the above types of constitutivity form a logical hierarchy. Absolute constitutivity entails essential-type constitutivity: if all flavour experiences require the functioning of some system $S$, then this is also the case for flavour experiences belonging to some type. Furthermore, essential constitutivity follows from essential-type constitutivity, as if all experiences belonging to some type cannot be obtained without the functioning of $S$, then there exists a flavour experience that cannot be obtained without the functioning of $S$. Finally, if the activities of $S$ are essentially constitutive, then they are also minimally constitutive. This is because, if there is a flavour experience that requires the functioning of $S$, then there is also a way of obtaining this experience involving the functioning of $S$.

In subsequent sections, I use empirical data concerning flavour perception to evaluate the constitutive status of the selected sensory systems.

\section{Olfaction and Gustation}

\subsection{Olfactory System}

There is wide consensus that the olfactory system is crucial for flavour perception. More specifically, what is crucial are mechanisms of retronasal olfaction which are stimulated by molecules coming from inside the mouth through the nasal cavity. Activities of retronasal olfaction mechanisms lead to flavour experiences in which some properties are attributed to entities localized in the mouth (Auvray \& Spence, 2008; Small et al., 2005). In opposition, orthonasal olfaction processes stimuli coming from outside the body and leads to experiences as of odours localized in the air around us. Such experiences are usually clearly distinct from flavour experiences. In most cases, the phenomenal aspects of flavour experiences occurring by virtue of retronasal olfaction are not easily introspectively distinguished from aspects provided by gustation, i.e. mechanisms processing information gathered by chemical receptors on the tongue (e.g., Marshall et al., 2006). Because of this, people are often unaware of the impact of the olfactory system on flavour perception.

The conviction that olfactory activities are crucial for flavour perception comes from the well-established observation that stimulating gustatory receptors leads only to taste experiences in which properties such as sweetness, sourness, or saltiness are presented (see Smith, 2013; Spence et al., 2014 for reviews). To obtain experiences presenting flavours such as strawberry, chocolate, or coffee, olfactory activities are needed. This common observation strongly suggests that the functioning of the olfactory system is essentially-type constitutive of flavour perception. This is because there are many commonly recognized types of 
flavour experiences which could not be obtained without the functioning of the olfactory system (Bartoshuk \& Duffy, 2011; Spence et al., 2014).

Of course, if olfactory system is essentially-type constitutive, then it is also essentially constitutive and minimally constitutive. However, a question remains, namely whether olfaction is also absolutely constitutive. One may believe that olfactory system should be regarded as absolutely constitutive, i.e. no flavour experience can be obtained without its functioning, because nearly every flavour stimulus influences the retronasal olfactory system in some way. However, I believe that such a claim is not without controversies.

Most importantly, there have been psychological studies specifically designed to investigate how blocking stimuli from activating olfactory receptors influences flavour perception. In such cases, the threshold for detecting tastants is higher (Mojet et al., 2005; Nagata el al., 2005). What is more, people make frequent mistakes regarding the categorization of presented stimuli (Simner et al., 2010). However, despite the fact that without olfactory activities flavour perception is significantly diminished, people are still able to have some experiences generated by combined functioning of, inter alia, gustatory, tactile, thermal, and trigeminal systems. The question is whether such experiences should be classified as flavour experiences. While they may be quite unlike the paradigmatic flavour experiences, I believe that there are some reasons for interpreting them as such.

As stated in the introductory section, flavour experiences are those which seem to occur in the mouth, are exteroceptive, multimodal, and are commonly referred to by using terms naming flavour-categories. The considered experiences which happen due to a combination of gustatory, tactile, thermal and trigeminal systems activities clearly satisfy the first three conditions: apparent location in the mouth, exteroceptivity and multimodality. Hence, the crucial question is whether such experiences not involving olfactory stimulation are likely to be named by flavour-related categorical terms. In fact, it has been observed that even experiences invoked by stimuli that solely influence gustatory and not olfactory receptors, like sucrose or table salt, are often described in typical terms naming flavour-categories (e.g. 'orangey' or 'fishy', see Simner et al., 2010, p. 566). Furthermore, while people with serious olfactory disorders often initially report a loss of the ability to taste, upon closer inspection, or after a period of time, they realize that they have some limited flavour perception which suggests that they are likely to use terms naming flavour-categories in describing their experiences (see Keller and Malsapina, 2013; Wrobel \& Leopold, 2004). In consequence, while the vast majority of flavour experiences require olfactory system functioning, it seems plausible that there are some flavour experiences which can occur without olfactory input. For instance, there may be a multimodal experience occurring due gustatory, thermal and tactile activities, which seems to happen in the mouth, ascribes properties to entities positioned in oral cavity, and is described by using a term such as 'orangey'. The occurrence of such experiences suggests the olfactory system is essential-type constitutive but not absolutely constitutive. 


\subsection{Gustatory System}

The second sensory system that is commonly treated as crucial for flavour perception is the gustatory system. This system gathers information from chemical receptors positioned in the oral cavity. Usually, five types of receptors are listed, which are associated with sweet, salt, bitter, sour, and umami taste sensations (see Auvray \& Spence, 2008). Sometimes this list is extended, as there are studies which suggest that activities of the gustatory system lead to 'metallic' sensations (Schiffman, 2000).

There is no doubt that functioning of the gustatory system is minimally constitutive of flavour perception, as there are many flavour experiences which can be obtained in a way that involves the functioning of gustation. Furthermore, it seems plausible that gustatory system is also essentially constitutive. Probably the most serious way of rejecting its essential status would be to claim that given the enormous impact of retronasal olfaction on flavour perception, all experiences usually involving gustatory activities may be evoked in an alternative way consisting in an appropriate stimulation of the olfactory, but not gustatory, system. Such a view may be motivated by two types of empirical results. First, it is well confirmed that olfactory activities are able to modify the aspects of flavour experiences that are usually associated with the functioning of the gustatory system (see Verhagen \& Engelen, 2006). The most popular example is the sweetness enhancement effect: a sweet gustatory stimuli is experienced as even more sweet when paired with certain odorants, for instance those responsible for an olfactory vanilla sensation (e.g., Labbe et al., 2006; Schifferstein \& Verlegh, 1996; Stevenson et al., 1999). Second, due to learned correlations between gustatory and olfactory stimuli, sensations evoked by odorants are often described using gustatory-related predicates (e.g., Prescott, 1999; Stevenson \& Boakes, 2004; Stevenson \& Tomiczek, 2007). For instance, odours are commonly described as sweet or sour.

Nevertheless, such interactions between the olfactory and gustatory systems do not justify the claim that gustatory system is not essentially constitutive of flavour perception. The sweetness enhancement and related phenomena show that olfactory activities may modify aspects of experiences that are usually associated with gustation, but they do not justify a stronger claim that all aspects introduced by gustation can be also obtained without gustatory activities by some appropriate stimulation of the olfactory system. Similarly, attributing gustatory-related predicates to sensations caused by odorants may suggest that some gustatory aspects of flavour experiences may also be introduced solely by virtue of olfactory activities. However, such effects are not sufficient to show that this is true of all gustatory-like qualities. In consequence, it seems that there are no strong empirically motivated reasons to refute the intuitive conviction that gustatory system is essentially constitutive of flavour perception.

A further question is whether gustatory system is also essentially-type constitutive of flavour perception. This would be the case if there were types of flavour experiences such that no exemplars of these types could be obtained without a functional gustatory system. Of course, it may be proposed that every gustatory-related phenomenal aspect, such as a sensation of sweetness, designates a type of flavour 
experience whose exemplars have precisely this aspect, and so it is trivially true that gustatory system is essentially-type constitutive.

However, when speaking about essential-type constitutivity I have in mind a stronger notion of flavour experience-types. My understanding is that in order to belong to the same type, flavour experiences must have phenomenal characters that are strongly associated, for instance by personal history or cultural learning, with eating members of a specific kind of external entity. For instance, a person may learn that experiences associated with eating strawberries are highly similar and group them within a common strawberry type. Furthermore, the strawberry type may also contain experiences caused by other things, like strawberry-flavoured ice creams or bubble gums, as they also cause experiences with phenomenal character that is recognised as similar to that associated with eating strawberries. On the other hand, it is less plausible that there is a specific class of entity that is strongly associated with generating sweet phenomenal character, as such character can occur when eating entities belonging to a huge variety of distinct kinds.

Given this stronger reading, one may propose that even types of flavour experiences that usually seem to be strongly associated with some gustatory aspects have exemplars that can occur due to olfactory activities without stimulating gustatory receptors. For instance, some authors suggest that while lemon-type experiences seem to be strongly associated with sourness, an exemplar of this type can also occur by virtue of a water solution combined with lemon-related odorants (see Murphy \& Cain, 1980). If such examples can be found in cases of all flavour-categories, then gustatory system is not essentially-type constitutive. However, there are serious doubts about whether experiences occurring in virtue of olfactory stimulation without gustatory stimulation should be treated as flavour experiences. A growing number of studies show that to obtain an experience which seems to happen within the mouth via olfactory stimulation, the stimulation of olfactory receptors must co-occur with the congruent gustatory simulation, i.e. such gustatory stimulation that commonly appears together with given retronasal stimulation and thus is highly associated with it (e.g., Lim \& Johnson, 2011, 2012). On the other hand, co-occurring tactile stimulation alone is insufficient for obtaining oral referral (see Spence, 2016). Given such results, it is plausible to assume that gustatory system is essentially-type constitutive of flavour perception.

If one accepts that gustatory system is essential-type constitutive, then the final question concerns the absolutely constitutive status of gustatory system functioning. Even if olfactory stimulation without congruent gustatory stimulation cannot provide flavour experiences, it can still be asked whether some flavour experiences may occur without both olfactory and gustatory activities. For instance, there may be experiences which occur in virtue of joint functioning of tactile, thermal and trigeminal stimulation. However, while such experiences seem to occur in the mouth, are exteroceptive and multimodal, it is unlikely that they are named by terms referring to flavour-categories as they are phenomenally distinct from usual flavour experiences. In fact, objects generating experiences of this type, such as ice cubes, are likely to be described as 'tasteless'. In consequence, their lack of flavour status makes it is plausible to accept that gustatory system is constitutive in the strongest, absolute sense. 


\section{Auditory and Visual Influences}

\subsection{Auditory System}

While according to an initial intuition, the activities of the auditory system are not of high importance for flavour perception, there is a significant body of evidence showing its influence on our experience of flavours (see Spence, 2014, 2015a). Of particular interest in the context of constitutivity are studies showing that auditory stimuli provide a relevant cue for experiencing food as being crisp (e.g., Velasco et al., 2018; Spence, 2014, 2015a, 2015b; Verhagen \& Engelen, 2006). Crackling sounds produced when crisp food is chewed are transferred through jaw bones and activate auditory mechanisms. In particular, it has been demonstrated that by providing appropriate auditory input through headphones, the experienced crispiness of eaten food can be enhanced (for instance, chips seem to be more crisp, Zampini \& Spence, 2004, see also Dematté et al., 2014 for an example with apples). In addition, in such experimental situations, participants perceive the provided sounds as coming not from the headphones, but from the food itself. This suggests that information processed by the auditory system is sensorily integrated with information processed by the gustatory, olfactory, and other systems.

I believe that the above results suggest not only that auditory activities causally modulate functioning of other sensory systems, but that they are actually minimally constitutive of flavour perception. For auditory activities to be minimally constitutive, there must be a flavour experience $F$ such that there is a way of obtaining $F$ which requires auditory activities. More technically, there must be a minimal set of conditions jointly sufficient for the occurrence of $F$ such that some conditions concerning the presence of synchronic auditory activities belong to this set. The information about crispiness of food may be acquired both by tactile and auditory modality. However, in the considered experiments only auditory information about crispiness changes as the texture of chips or apples remains the same. Nevertheless, despite the sameness of tactile information, the perception of crispiness varies significantly due to changing auditory input. Furthermore, it is unlikely that in the considered cases, the crispiness perception changes solely because the auditory system modifies the functioning of the tactile system. This is because participants do not experience crackling sounds coming from headphones, hence judging crispiness relying merely on the perceived texture; instead they believe that crackling sounds come from the eaten food. This suggests that the functioning of the auditory system directly contributes to an assessment of crispiness and does not merely influence tactile aspects of flavour experience.

In consequence, there is a set of conditions jointly sufficient for a certain experience of crispiness $F$ which contains some conditions regarding auditory activities and some conditions regarding tactile activities. Furthermore, this set is minimal, in the sense that removing conditions regarding auditory activities would, ceteris paribus, result in a set of conditions which are no longer sufficient for the presence of a crispiness experience, or would result in a different 
experience than $F$, with a lower level of crispiness. This is because in the considered situation, tactile activities alone would not lead to the experience $F$ but at best to some experience in which crispiness is less pronounced. Hence, there is a flavour experience $F$ such that there is a way of obtaining $F$ which requires functioning of the auditory system, and so audition is minimally constitutive of flavour perception.

The investigations regarding crispiness are of particular importance, as other auditory influences on flavour perception commonly mentioned in the literature, like modification of intensity and gustatory aspects of food by environmental noise (see Spence, 2014), can be plausibly interpreted as merely causal influences. In those cases, auditory activities modulate functioning of the gustatory, olfactory, and other systems, and as a result some flavour experience $F$ occurs. However, the minimal set of conditions jointly sufficient for $F$ does not contain auditory activities, since a joint occurrence of gustatory, olfactory, and other systems would be sufficient for $F$ 's occurrence. The role of the auditory system is only to causally evoke those gustatory, olfactory, and other activities whose combination is sufficient for the experience $F$.

It should be noted that 'minimal constitutivity' is a qualitative category such that functioning of a sensory system may be minimally constitutive for flavour perception even if there is only a single experience $F$ such that there is a way of obtaining it which requires activities of the considered system. An additional, quantitative notion of 'scope' can be introduced to account for the number of experiences for which functioning of a given sensory experience is constitutive. By using it one can state that while functioning of the auditory system is constitutive, according to the current state of the art its constitutive character may have very limited scope.

On the other hand, it is less plausible that the auditory system should be treated as essentially constitutive. In fact, there are studies suggesting that information about the crispiness of food obtained by the auditory system is redundant and can be replaced by tactile information (see Delwiche, 2004; Spence, 2015a for a review). Crisp food breaks into pieces as a result of chewing and produces vibrations that are detected by tactile mechanisms and allow the crispiness to be assessed. Hence, it may be the case that while there is a crispiness experience $F$ for which there is a way of obtaining it which requires both some auditory activities $A$ and tactile activities $T$, there may be another way of obtaining $F$ such that $F$ happens due to some distinct tactile activities $T 1$ and without auditory input. For instance, possibly the same crispiness experience can occur due to a combination of some auditory activities and moderate tactile activities as well as due to strong tactile activities despite lack of auditory stimulation. If this is the case, then the auditory system is not essentially constitutive of flavour perception as there are no experiences that can only be obtained with auditory system functioning.

Overall, it seems reasonable to state that auditory system is merely minimally constitutive of flavour experiences. Nevertheless, it is less likely that it is also essentially constitutive. 


\subsection{Visual System}

There seem to be a consensus among flavour scientists that there are certain ways in which vision influences flavour perception (e.g., Delwiche, 2004; Shankar et al., 2010a, 2010b; Spence, 2015b, 2015c; Verhagen \& Engelen, 2006). In particular, while it is less clear whether visual activities modify the perceived intensity of flavours, it seems well-established that they help in flavour identification, understood as recognizing the category, like 'strawberry', to which a flavour belongs (see Spence et al., 2010 for a review). However, it is also widely believed that visually formed flavour-related expectations play an important role in visual influences on flavour perception (see Shankar et al., 2010a, 2010b; Spence et al., 2010). The assessment of the exact role of such expectations is crucial for determining whether visual system is constitutive of flavour perception. In particular, there seem to be three major interpretations concerning the role of visually formed flavour-expectations:

(1) Visual activities lead to certain expectations but do not influence the phenomenal character of flavour experiences (see Carvalho et al., 2017; Wang et al., 2017 for studies suggesting such a view). For instance, when given a yellow beverage, a person may expect it to be sourer, but the taste-component of the overall experience of flavour is not modified by these expectations.

(2) While visual activities do not directly influence the phenomenal character of flavour experiences, such influence is mediated by the visually formed expectations. For instance, the flavour of a yellow beverage may actually seem sourer due to the top-down influence of visually formed expectations.

(3) Visual activities are able, at least in some cases, influence the phenomenal character of flavour experiences without or independent of the influences of expectations (see Hyman, 1983; Johnson \& Clydesdale, 1982; Maga, 1974 for studies suggesting such a view). In this case, a yellow beverage may actually seem sourer in virtue of visual activities.

If the first option is true of all visual influences on flavour perception, then it is obvious that visual system is not constitutive. Furthermore, even if the second option is true, and influences on flavour perception are caused by visually formed expectations, the visual system would not be even minimally constitutive of flavour perception. This is because for system to be minimally constitutive, there must be an experience $F$ with a minimal set of jointly sufficient occurrence conditions such that some of these conditions concern the presence of the activities of the considered system. However, in the case discussed, a sufficient set of occurrence conditions, including conditions regarding both visual activities and expectations, is not minimal. Conditions belonging to a smaller set, which does not contain conditions regarding visual activities but those regarding expectations, would still be a set of jointly sufficient conditions of $F$ 's occurrence if only the relevant expectations could be activated without visual input (e.g., by virtue of some propositional information delivered by auditory speech stimuli). 
However, if the third option is the correct one, and there are cases in which visual activities modify the flavour experiences independent of expectations, then minimal constitutivity can be attributed to functioning of the visual system. I believe that the currently available data do not allow us to decisively state which of the above options is true. In consequence, it is possible that visual system is minimally constitutive of flavour perception, but one can also have justified doubts whether it is constitutive at all. On the other hand, there are no data suggesting that visual system can be essentially constitutive. The functioning of visual mechanisms does not introduce any novel aspects of flavour experiences, but merely modifies those which occur by virtue of gustatory, olfactory, and tactile activities (Shankar et al., 2010a, 2010b; Spence, 2015b, 2015c; Verhagen \& Engelen, 2006). There are no strong reasons to believe that any of these visually modified flavour experiences couldn't be re-created without visual input by appropriately arranging stimuli connected with other sensory systems.

\section{Conclusions}

By applying the theoretical framework proposed in section three and relying on current empirical findings regarding flavour perception, it has been possible to assess the constitutive status of various sensory systems whose activities are likely to influence how we experience flavours. In some cases the current state of knowledge did not allow for decisively choosing a single interpretation of the constitutive status of a given type of sensory system. Nevertheless, in all considered cases one may formulate constraints that determine the most likely interpretations.

In the case of the olfactory system, it is clear that its functioning is essential-type constitutive. However, there seem to be experiences, created by a combination of gustatory, thermal, tactile and trigeminal stimulation, which satisfy the characteristic of flavour experiences without involving olfactory activities. While such experiences are rather rare, their presence is sufficient to reject the claim that olfactory system is absolutely constitutive.

On the other hand, the gustatory system is likely to be absolutely constitutive of flavour perception. This is because the olfactory stimulation without congruent gustatory stimulation does not lead to experiences apparently happening in the mouth, and experiences obtained merely due to combination of tactile, thermal, and trigeminal activities are unlikely to be named by using terms referring to flavour-categories.

In considerations regarding auditory systems, I argued that there are good reasons to postulate that auditory system is minimally constitutive of flavour perception. This is because there are certain specific flavour experiences, such as experiences of crispiness, which can be obtained in a way that requires some functioning of the auditory system. However, it less likely it is also essentially constitutive as there are data suggesting that auditory activities can be replaced by tactile activities.

Visual system is the only type for which there are serious reasons to believe that it is simply not constitutive of flavour perception. There is a variety of data suggesting that visual activities at least sometimes modify flavour experiences. However, it is not obvious whether visual activities are able to directly influence flavour 
experiences, or whether their influence is always mediated by expectations, i.e. vision activates certain expectations concerning flavours and those expectations subsequently change the phenomenal character of flavour experiences. If the first option is true, then visual system is minimally constitutive. If the second is the case, it is not constitutive at all.

Acknowledgements The author would like to thank the anonymous reviewers for comments concerning the paper. The first idea concerning the paper came to my mind as a result of discussions during the Models of Mechanisms in Computational Neuroscience workshop at Witten-Herdecke University. The early version of the paper was presented during SemDok seminar at Jagiellonian University, CLMPST 2019 conference in Prague, ESPP 2019 conference in Athens, and Riga Symposium on Senses 2019.

Funding The work was supported by the National Science Center (Poland) grant 2016/20/S/HS1/00090.

\section{Declarations}

I comply with the Ethical Standards of "Erkenntnis".

Conflict of interest The author declares that he has no conflict of interest.

Human or Animal Rights Informed consent and animal welfare: The research did not involve human participants or animals.

Open Access This article is licensed under a Creative Commons Attribution 4.0 International License, which permits use, sharing, adaptation, distribution and reproduction in any medium or format, as long as you give appropriate credit to the original author(s) and the source, provide a link to the Creative Commons licence, and indicate if changes were made. The images or other third party material in this article are included in the article's Creative Commons licence, unless indicated otherwise in a credit line to the material. If material is not included in the article's Creative Commons licence and your intended use is not permitted by statutory regulation or exceeds the permitted use, you will need to obtain permission directly from the copyright holder. To view a copy of this licence, visit http://creativecommons.org/licen ses/by/4.0/.

\section{References}

Arvisenet, G., Guichard, E., \& Ballester, J. (2016). Taste-aroma interaction in model wines: Effect of training and expertise. Food Quality \&amp; Preference, 52, 211-221.

Auvray, M., \& Spence, C. (2008). The multisensory perception of flavor. Consciousness and Cognition, 17, 1016-1031.

Bartoshuk, L. M., \& Duffy, V. B. (2011). Chemical senses. In G. Greenberg \& M. M. Haraway (Eds.), Comparative psychology: A handbook (pp. 282-289). Routledge.

Bartoshuk, L. M., Duffy, V. B., Lucchina, L. A., Prutkin, J., \& Fast, K. (1998). PROP (6-n-propylthiouracil) supertasters and the saltiness of $\mathrm{NaCl}$. Annals of the New York Academy of Sciences, 855, 793-796.

Baumgartner, M., \& Gebharter, A. (2015). Constitutive relevance, mutual manipulability, and fat-handedness. British Journal for the Philosophy of Science, 67(3), 731-756.

Bennett, K. (2011). Construction area (no hard hat required). Philosophical Studies, 154, 79-104.

Carvalho, F. R., Moors, P., Wagemans, J., \& Spence, C. (2017). The influence of color on the consumer's experience of beer. Frontiers in Psychology. https://doi.org/10.3389/fpsyg.2017.02205

Couch, M. B. (2011). Mechanisms and constitutive relevance. Synthese, 183, 375-388.

Craver, C. (2007). Constitutive explanatory relevance. Journal of Philosophical Research, 32, 3-20. 
Delwiche, J. (2004). The impact of perceptual interactions on perceived flavor. Food Quality and Preference, $15,137-146$.

Dematté, M. L., Pojer, N., Endrizzi, I., Corollaro, M. L., Betta, E., Aprea, E., Charles, M., Biasioli, F., Zampini, M., \& Gasperi, F. (2014). Effects of the sound of the bite on apple perceived crispness and hardness. Food Quality and Preference, 38, 58-64.

Deroy, O., Spence, C., \& Noppeney, U. (2016). Causal metacognition: Monitoring uncertainty about the causal structure of the world. Trends in Cognitive Sciences, 20, 736-747.

Gebharter, A. (2016). Uncovering constitutive relevance relations in mechanisms. Philosophical Studies, 174(11), 2645-2666.

Gillett, C. (2013). Constitution, and multiple constitution, in the sciences: Using the neuron to construct a starting framework. Minds \&amp; Machines, 23, 309-337.

Harbecke, J. (2010). Mechanistic constitution in neurobiological explanations. International Studies in the Philosophy of Science, 24(3), 267-285.

Hyman, A. (1983). The influence of color on the taste perception of carbonated water preparations. Bulletin of Psychonomic Society, 21, 145-148.

Johnson, J., \& Clydesdale, F. (1982). Perceived sweetness and redness in colored sucrose solutions. Journal of Food Science, 47, 747-752.

Kaiser, M. I., \& Krickel, B. (2016). The metaphysics of constitutive mechanistic phenomena. The British Journal for the Philosophy of Science, 68(3), 745-779.

Keeley, B. J. (2002). Making sense of the senses. Journal of Philosophy, 99(1), 5-28.

Keller, A., \& Malaspina, D. (2013). Hidden consequences of olfactory dysfunction: A patient report series. BMC Ear Nose and Throat Disorders, 12, 8. https://doi.org/10.1186/1472-6815-13-8

Kirchhoff, M. (2013). Extended cognition \& constitution: Reevaluating the constitutive claim of extended cognition. Philosophical Psychology, 27(2), 258-283.

Kirchhoff, M. (2015). Extended cognition \& the causal-constitutive fallacy: In search for a diachronic and dynamical conception of constitution. Philosophy and Phenomenological Research, 90(2), 320-360.

Krickel, B. (2018). Saving the mutual manipulability account of constitutive relevance. Studies in History and Philosophy of Science, 68, 58-67.

Labbe, D., Damevin, L., Vaccher, C., Morgenegg, C., \& Martin, N. (2006). Modulation of perceived taste by olfaction in familiar and unfamiliar beverages. Food Quality and Preference., 17(7-8), 582-589.

Leuridan, B. (2012). Three problems for the mutual manipulability account of constitutive relevance in mechanisms. British Journal for the Philosophy of Science, 63, 399-427.

Lim, J., \& Johnson, M. B. (2011). Potential mechanisms of retronasal odor referral to the mouth. Chemical Senses, 36, 283-289.

Lim, J., \& Johnson, M. (2012). The role of congruency in retronasal odor referral to the mouth. Chemical Senses, 37, 515-521.

Lycan, W. G. (2018). What does taste represent? Australasian Journal of Philosophy, 96(1), $28-37$.

Maga, J. (1974). Influence of colors on taste tresholds. Chemical Senses, 1(1), 115-119.

Marshall, K., Laing, D. G., Jinks, A., \& Hutchinson, I. (2006). The capacity of humans to identify components in complex odor-taste mixtures. Chemical Senses, 31(6), 539-545.

Mojet, J., Köster, E. P., \& Prinz, J. F. (2005). Do tastants have a smell? Chemical Senses, 30, 9-21.

Murphy, C., \& Cain, W. (1980). Taste and olfaction: Independence vs interaction. Physiology and Behavior, 24, 601-605.

Nagata, H., Dalton, P., Doolittle, N., \& Breslin, P. A. S. (2005). Psychophysical isolation of the modality responsible for detecting multimodal stimuli: A chemosensory example. Journal of Experimental Psychology: Human Perception \&amp; Performance, 31, 101-109.

Prescott, J. (1999). Flavour as a psychological construct: Implications for perceiving and measuring the sensory qualities of foods. Food Quality and Preference, 10, 349-356.

Prescott, J. (2015). Multisensory processes in flavour perception and their influence on food choice. Current Opinion in Food Science, 3, 47-52.

Reed, D. R., \& Knaapila, A. (2010). Genetics of taste and smell: Poisons and pleasures. Progress in Molecular Biology Translational Science, 94, 213-240.

Rolls, E. T. (1997). Taste and olfactory processing in the brain and its relation to the control of eating. Critical Reviews in Neurobiology, 11, 263-287.

Schifferstein, H. N. J., \& Verlegh, P. W. J. (1996). The role of congruency and pleasantness in odorinduced taste enhancement. Acta Psychologica, 94, 87-105.

Schiffman, S. S. (2000). Taste quality and neural coding: Implications from psychophysics and neurophysiology. Physiology and Behavior, 69, 147-159. 
Shankar, M. U., Levitan, C. A., \& Spence, C. (2010a). Grape expectations: The role of cognitive influences in color-flavor interactions. Consciousness and Cognition, 19, 380-390.

Shankar, M., Simons, C., Shiv, B., Levitan, C., McClure, S., \& Spence, C. (2010b). An expectationsbased approach to explaining the influence of color on odor identification: The influence of degree of discrepancy. Attention Perception Psychophysics, 72, 1981-1993.

Simner, J., Cuskley, C., \& Kirby, S. (2010). What sound does that taste? Cross-modal mapping across gustation and audition. Perception, 39, 553-569.

Simons, C. T., \& Noble, A. C. (2002). Challenges for the sensory sciences from the food and wine industries. Nature Reviews, 4, 599-605.

Small, D. M. (2012). Flavor is in the brain. Physiology \&amp; Behavior, 107, 540-552.

Small, D. M., Gerber, J. C., Mak, Y. E., \& Hummel, T. (2005). Differential neural responses evoked by orthonasal versus retronasal odorant perception in humans. Neuron, 47(4), 593-605.

Small, D. M., \& Prescott, J. (2005). Odor/taste integration and the perception of flavor. Experimental Brain Research, 166, 345-357.

Smith, B. C. (2007). Questions of taste. The philosophy of wine. Oxford University Press.

Smith, B. C. (2013). The nature of sensory experience: The case of taste and tasting. Phenomenology and Mind, 4, 212-227.

Spence, C. (2014). Noise and its impact on the perception of food and drink. Flavour. https://doi.org/10. 1186/2044-7248-3-9

Spence, C. (2015a). Eating with our ears: assessing the importance of the sounds of consumption on our perception and enjoyment of multisensory flavour experiences. Flavour. https://doi.org/10.1186/ 2044-7248-4-3

Spence, C. (2015b). Multisensory flavor perception. Cell, 161, 24-35.

Spence, C. (2015c). On the psychological impact of food colour. Flavour. https://doi.org/10.1186/ s13411-015-0031-3

Spence, C. (2016). Oral referral: On the mislocalization of odours to the mouth. Food Quality \&amp; Preference, 50, 117-128.

Spence, C., Levitan, C., Shankar, M. U., \& Zampini, M. (2010). Does food color influence taste and flavor perception in humans? Chemosensory Perception, 3, 68-84.

Spence, C., Smith, B., \& Auvray, M. (2014). Confusing tastes with flavours. In D. Stokes, M. Matthen, \& S. Briggs (Eds.), Perception and its modalities (pp. 247-276). Oxford University Press.

Spence, C., \& Zampini, M. (2006). Auditory contributions to multisensory product perception. Acta Acustica, 92, 1009-1025.

Stevenson, R. J. (2009). The psychology of flavour. Oxford University Press.

Stevenson, R. J. (2012). The role of attention in flavour perception. Flavour. https://doi.org/10.1186/ 2044-7248-1-2

Stevenson, R. J. (2014). Object concepts in the chemical senses. Cognitive Science, 38(2014), 1360-1383.

Stevenson, R. J., \& Boakes, R. A. (2004). Sweet and sour smells: the acquisition of taste-like qualities by odors. In G. Calvert, C. Spence, \& B. Stein (Eds.), Handbook of multisensory processing (pp. 69-83). MIT Press.

Stevenson, R. J., Prescott, J., \& Boakes, R. A. (1999). Confusing tastes and smell: How odours can influence the perception of sweet and sour tastes. Chemical Senses, 24, 627-635.

Stevenson, R. J., \& Tomiczek, C. (2007). Olfactory-induced synesthesias: A review and model. Psychological Bulletin, 133(2), 294-309.

Titchener, E. B. (1909). A textbook of psychology, Part 1. Macmillan.

Velasco, C., Obrist, M., Petit, O., \& Spence, C. (2018). Multisensory technology for flavor augmentation: A mini review. Frontiers in Psychology. https://doi.org/10.3389/fpsyg.2018.00026

Verhagen, J. V. (2007). The neurocognitive bases of human multimodal food perception: Consciousness. Brain Research Reviews, 53, 271-286.

Verhagen, J. V., \& Engelen, L. (2006). The neurocognitive bases of human multimodal food perception: Sensory integration. Neuroscience and Biobehavioral Reviews, 30, 613-650.

Wan, X., Woods, A. T., van den Bosch, J. F. F., McKenzie, K. J., Velasco, C., \& Spence, C. (2014). Cross-cultural differences in crossmodal correspondences between basic tastes and visual features. Frontiers in Psychology. https://doi.org/10.3389/fpsyg.2014.01365

Wang, Q. J., Carvalho, F. R., Persoone, D., \& Spence, C. (2017). Assessing the effect of shape on the evaluation of expected and actual chocolate flavour. Flavour. https://doi.org/10.1186/ s13411-017-0052-1

Wasserman, R. (2004). The constitution question. Nous, 38, 693-710. 
Wilson, R. A. (2007). A puzzle about material constitution \& how to solve it: Enriching constitution views in metaphysics. Philosophers Imprint, 7(5), 1-20.

Wrobel, B. B., \& Leopold, D. A. (2004). Clinical assessment of patients with smell and taste disorders. Otolaryngologic Clinics of North America, 37(6), 1127-1142.

Ylikoski, P. (2013). Causal and constitutive explanation compared. Erkenntnis, 78(2), 277-297.

Zampini, M., \& Spence, C. (2004). The role of auditory cues in modulating the perceived crispness and staleness of potato chips. Journal of Sensory Studies, 19(5), 347-363.

Publisher's Note Springer Nature remains neutral with regard to jurisdictional claims in published maps and institutional affiliations. 